\title{
Visual speech information for face recognition
}

\author{
LAWRENCE D. ROSENBLUM, DEBORAH A. YAKEL, NASER BASEER, ANJANI PANCHAL, \\ BRYNN C. NODARSE, and RYAN P. NIEHUS \\ University of California, Riverside, California
}

\begin{abstract}
Two experiments test whether isolated visible speech movements can be used for face matching. Visible speech information was isolated with a point-light methodology. Participants were asked to match articulating point-light faces to a fully illuminated articulating face in an XAB task. The first experiment tested single-frame static face stimuli as a control. The results revealed that the participants were significantly better at matching the dynamic face stimuli than the static ones. Experiment 2 tested whether the observed dynamic advantage was based on the movement itself or on the fact that the dynamic stimuli consisted of many more static and ordered frames. For this purpose, frame rate was reduced, and the frames were shown in a random order, a correct order with incorrect relative timing, or a correct order with correct relative timing. The results revealedbetter matching performance with the correctly ordered and timed frame stimuli, suggesting that matches were based on the actual movement itself. These findings suggest that speaker-sh Visual speech style can provide information for face matching. information for face recognition
\end{abstract}

Historically, recognizing who is speaking

being said have been considered to be separate When speech is listened to, voice and phonetic have been thought to use separate information, and neurophysiology(Assal, Aubert, \& Buttet, 1 Zander, Kremin, \& Buttet, 1976; Bricker \& Pruzansky, 1966; Van Lancker, Cummings, Kreiman, \& Dobkin, 1988). When speech is seen, the functions of face recognition and lipreading are also considered to be distinct in information, operations, and neurophysiology (Bruce \& Young, 1986; Campbell, Landis, \& Regard, 1986; Ellis, 1989; Fodor, 1983; Liberman \& Mattingly, 1985). However, recent research on both modalities has challenged these assumed independencies(for reviews, see Nygaard, Sommers, \& Pisoni, 1994; Remez, Fellowes, \& Rubin, 1997; Rosenblum, Yakel, \& Green, 2000; Schweinberger \& Soukup, 1998; Yakel, Rosenblum, \& Fortier, 2000). Regarding auditory speech, there is evidence that voice information can facilitate linguistic recovery in the contexts of word naming and identification in noise (Mullennix, Pisoni, \& Martin, 1989; Nygaard et al., 1994), recognition memory (Craik \& Kirsner, 1974; Palmeri, Goldinger, \& Pisoni, 1993), implicit memory (Church \& Schacter, 1994), and form-based priming (Saldaña \& Rosenblum, 1994).

For visual speech, analogous findings have shown some dependence of visual speech perception on face familiar-

This research was supported by NSF Grant SBR-9617047 awarded to the first author. We gratefully acknowledge the assistance of Neley Hakimi, as well as the helpful comments the UCR Cognitive Science group. D.A.Y. is currently at Orange Coast College, Department of Psychology, N.B. is currently at Illinois Institute of Technology, and A.P. is currently at Northern Illinois University. Correspondence concerning this article should be addressed to L. D. Rosenblum, Department of Psychology, University of California, Riverside, CA 92521 (e-mail: rosenblu @ citrus.ucr.edu).

are familiar with the faces. Walker, Bruce, and O'Malley (1995) found that participants who were familiar with the faces of the speakers portrayed in the stimuli were less susceptible to the McGurk effect (McGurk \& McDonald, 1976) when an incongruent voice was paired with a familiar face. Finally, Sheffert and Fowler (1995) have found that visual speaker information can be retained along with audiovisually presented words. These findings support the notion that familiarity with a speaker's face facilitates visual speech perception.

Other recent evidence supports the relation between visual speech and face perception. Stimulus manipulations known to disproportionately disrupt face recognition have also been shown to inhibit visual speech perception (see Rosenblumet al., 2000, for a review). This has been found of inverted faces (Green, 1994; Jordan \& Bevan, 1997; Massaro $\&$ Cohen, 1996), as well as of faces in which the mouth alone is inverted (i.e., the Margaret Thatcher effect; Rosenblum et al., 2000; Thompson, 1980). The fact that both functions are influenced by these specific manipulations has been interpreted as support for the involvement of common information or processes (e.g., Jordan \& Bevan, 1997; Rosenblum et al., 2000).

For auditory speech, recent findings have also challenged the assumed distinction in the information used for phonetic and voice recognition. Specifically, Remez and his colleagues (Remez et al., 1997) have shown that isolated phonetic information (in the form of sinewave speech) can be used for both phonetic and speaker recovery. Analogously, it could be that common information can 
be used for both face recognition and visual speech perception purposes (see also Rosenblum et al., 2000). Usually, the visual attributes for face recognition and visual speech perception are considered to be very different. For face recognition, information is thought to consist of the shape and configuration of the facial features, as well as skin tone, hair, and the overall shape of the face (see Bruce, 1988 , for a review). In contrast, the features for visual speech perception are often construed as open, closed, or rounded lips, and visible teeth (e.g., McGrath, 1985; Montgomery \& Jackson, 1983; Petajan, 1984; Summerfield \& McGrath 1984) and/or the dynamic attributes of articulation (e.g., Barber, Vitkovitch, Wallbott, Ellgring, \& Kotthaus, 1990; Goldschen, 1993; Green \& Gerdman, 1995; Rosenblum \& Saldaña, 1996; Vitkovitch \& Barber, 1994; and see Rosenblum \& Saldaña, 1998, for a review).

Work in our laboratory has revealed that isolated kinematic features of an articulating face can be informative for visual speech perception (e.g., Rosenblum, Johnson, \& Saldaña, 1996; Rosenblum \& Saldaña, 1996). For these demonstrations, a point-light technique is implemented in which fluorescent dots are placed on the lips, teeth, tongue, chin, and cheeks of a speaker. The speaker is filmed articulating in the dark so that the resultant displays are seen as bright, moving dots against a black background. Our results show that facial point-light displays enable observers to distinguish vowels, consonants, words, and sentences, as well as to identify many consonantal visemes (Rosenblum, Johnson, \& Mahmood, 2001). We have also shown that these displays can improve the speech reception thresholds of sentences in noise (Rosenblum et al., 1996) and can influence auditory speech in an integration paradigm (Rosenblum \& Saldaña, 1996). Importantly, we have found that when our images are frozen in time, observers cannot identify them as faces, indicating that our stimuli do not contain recognizable static facial feature information. This research suggests that time-varying information could account for a large proportion of the variance for visual speech perception.

There is also evidence that dynamic visual information can facilitate face recognition (Christie \& Bruce, 1998; Knight \& Johnston, 1997; Lander, Christie, \& Bruce, 1999; Lander, Rosenblum, \& Bruce, 2001). In one example, Lander et al. (1999) used a number of image degradation techniques to distort pictorial face information. These techniques involved image inversion and thresholding, which relegates all image components to either black or white. Lander and her colleagues found that participants were significantly better at recognizing the faces when these degraded images were shown moving than when the images were presented as groups of static frames.

The point-light technique has also been used to demonstrate the salience of dynamic information for nonspeech aspects of face perception. There is evidence that observers can recover emotional expression, gender, and age-related "person" qualities from point-light displays (Bassili, 1978; Berry, 1990, 1991; Bruce \& Valentine, 1988). Most relevant is work conducted by Bruce and Valentine showing that point-light stimuli can provide information for identifying faces. Point-light faces were filmed producing different emotional expressions (frowning, smiling, surprised) as well as various "rigid" head motions (nodding, shaking, side-to-side rocking). The displays were then shown to participants who were familiar with the faces. The results showed that the participants could identify the faces at better than chance levels when the displays were shown moving, but not when the displays were shown statically. Overall, however, the participants were not very accurate at the task.

Although evidence demonstrates that face recognition information can be conveyed by dynamic displays, there is no direct evidence that dynamic articulatory information is salient for these purposes. Besides the work in our laboratory, facial point-light experiments have not included points on the speech articulators and have rarely tested linguistic material. This is certainly the case for the Bruce and Valentine (1988) study. Also, although many of the actors used in the stimuli of the Lander et al. (1999) study were shown talking, they were also shown moving their heads and upper bodies in various ways. Thus, the stimuli in past studies have not provided tests of whether visible articulatory information can be used for face recognition purposes.

The following experiments are an initial test of whether isolated visual speech information can be used for face recognition. In this sense, the experiments are a visual analogue of Remez et al.'s (1997) test of common auditory primitives for speaker and speech perception. For our experiments, a point-light technique was implemented to isolate visual speech. Although points were applied to various locations on the face, our actors were instructed only to speak, and their heads were made immobile. For this first test of these stimuli, a matching-to-sample (XAB) procedure was used, for which participants were asked to match each articulating point-light face to the same (articulating) face fully illuminated (Remez et al., 1997). Although other dynamic face research has tested straight identification judgments (e.g., Bruce \& Valentine, 1988; Lander et al., 1999), a matching test might allow observers to make use of the more subtle articulatory movements contained in the stimuli. For Experiment 1, the stimuli were also shown under static conditions, to assess whether successful matches might be based on whatever static information remained in the point-light displays (cf. Bruce \& Valentine, 1988; Johansson, 1973). If visible kinematic speech information is informative for face recognition, observers should be able to make matches with the dynamic point-light stimuli at better than chance levels and more successfully than with the static stimuli.

\section{EXPERIMENT 1}

\section{Method}

Participants. Twenty undergraduates were given class credit for their participation. They were all native English speakers and had (self-reported) normal vision and hearing. Their ages ranged from 18 to 24 years. 
Materials. Ten native American-English speakers (5 male and 5 female) were videotaped with a Panasonic PVS350 camcorder from a distance of $5 \mathrm{ft}$. The speakers' heads were secured by a wooden frame to inhibit head movement and to add background points for the point-light images (see below). In the recorded image, each speaker's entire head was visible against this background.

The speakers were asked to repeatedly articulate the sentence "The football game is over." This sentence was chosen because it has been found to be particularly easy to lip-read under both fully illuminated face and point-light conditions (Rosenblum et al., 1996). For their articulations, the speakers were told to maintain a normal and even intonation, tempo, and vocal intensity. At the same time, all the speakers were encouraged to produce the sentence with a duration of $2-3 \mathrm{sec}$. The speakers were not informed of the purpose of the recordings.

The sentences were recorded under two lighting conditions. For the fully illuminated face stimuli, the speakers were recorded with full overhead (fluorescent) lighting, along with a single incandescent spotlight situated about $4 \mathrm{ft}$ in front of the face. No special effort was made to illuminate the inside of the mouth; however, the spotlight was directed so that minimal shadowing occurred. For the pointlight stimuli, the speakers were illuminated with two black light (fluorescent) 24 -in., $10-\mathrm{W}$ bulbs held vertically $3 \mathrm{ft}$ away and at a $45^{\circ}$ angle to the side/front of the face; no other lighting was used. In order to hide any specularities provided by mouth moisture, speakers' tongues were painted black with food coloring, and their teeth were painted black with Mehron ToothFX tooth paint. Construction paper dots were made with a 3-mm hole punch and were painted with yellow Wildfire ultraviolet-sensiti ve fluorescent paint in brilliant yellow. These dots were applied to the face with medical adhesive (on the face) and dental adhesive (on the teeth and tongue). (Black coloring was not needed on the face itself.) The dots were small enough that they did not interfere with the speakers' articulations. This lighting and dot technique produced recorded images in which only the dots and their movements could be seen.

Thirty dots were placed on each speaker's face. The selection of dot positions was based on three considerations. First, positions were chosen so as to convey good visual speech information. Previous research has shown that dots placed on the lips, teeth, and tongue are particularly useful for conveying visual speech information (Rosenblum et al., 1996; Rosenblum \& Saldaña, 1996). Accordingly, in addition to various locations on the cheeks, jaw, and forehead (15 total), dots were placed on the front teeth (4), tongue tip (1), and at various locations on the lips (10).

Second, point positions were chosen so as not to convey static facial structure information. In order to test whether isolated articulatory information was salient for face recognition, it was important that the images not convey speaker-specific information when shown statically. To accomplish this goal, many of the points were placed on random positions on the facial features (lips, cheeks, chin, nose, forehead), and the random placement was different for each speaker. This precluded structural comparisons between point-light images, which would be available if the same point positions were chosen for all the speakers. To further preclude the use of the points to convey static information, the speakers (when in the point-light condition) were videotaped with their faces positioned in a black wooden frame that was covered with a random array of points.

Third, pilot studies revealed that participants could use the speakerspecific pattern of points to make static matches, using a process of elimination. For our stimulus-to-sample matching procedure, each fully illuminated face was shown with its matched point-light face repeatedly and with the nonmatched point-light faces just twice. Because the random pattern of points was distinctive for each speaker, pilot participants reportedly learned to match a particular pattern to the fully illuminated face it was matched with most often. This allowed the participants to perform successful static matches based spuriously on the patterns of points. To preclude this strategy, nine different random point-light patterns were recorded for each speaker. This allowed for no specific point-light pattern to be seen more than twice during the experiment.

For each recorded speaker, specific test sentences (fully illuminated and point-light) were chosen on the basis of general clarity of articulation and regularity in duration, and no explicit comparisons across sentences for a given speaker were made. The average duration of the utterances was 3 sec.

The recorded stimuli were then digitally captured onto computer for purposes of editing and establishing presentation orderings. The resultant stimuli were then recorded onto videotape for presentation to the participants. Two presentation tapes were produced: one for the dynamic condition and one for the static condition. For both tapes, 100 different utterances were used: for each of 10 speakers, 1 fully illuminated and 9 point-light utterances. For both tapes, the utterances were arranged so that a fully illuminated utterance of a given speaker was presented first, followed by two point-light utterances, one of which was of the speaker portrayed in the fully illuminated image and the other of which was of one of the other nine speakers (XAB stimulus arrangement). Each speaker's fully illuminated image was combined with each of the other nine speakers' images twice: once so that the first point-light image matched the fully illuminated image, and once so that the second point-light image matched the fully illuminated image. This produced a total of 180 randomized triads (10 participants $\times 9$ matches $\times 2$ orderings). The sentences were each $3 \mathrm{sec}$ long, with interstimulus intervals (ISIs) of $1 \mathrm{sec}$ between utterances in a triad and $3 \mathrm{sec}$ between triads.

For the dynamic stimulus tape, the sentences were played in their entirety. For the static stimulus tape, a single frame was presented from each of the utterances (Bruce \& Valentine, 1988; Johansson, 1973). Frames were chosen to depict a neutral articulatory position (slightly open mouth) for both the fully illuminated and the pointlight recordings. These static frames were each presented for $3 \mathrm{sec}$, and were organized in triads in the way described above. The same triad ordering was used for the dynamic and the static stimulus tapes.

Procedure. Ten of the participants were randomly assigned to the dynamic condition and the other 10 participants were assigned to the static condition. The participants were tested individually and were seated at a table in a $11 \times 9 \mathrm{ft}$ room facing a 20-in. Panasonic Color Video Monitor (CT-2010Y) at a distance of $5 \mathrm{ft}$. The monitor was adjusted to maximize the contrast for the point-light images, and the color was turned off. The participants were instructed to attend carefully to the video monitor during each set of three stimuli. They were asked to watch each full triad, and to judge whether the fully illuminated face matched the first or the second point-light face (XAB procedure). They were asked to indicate their choice by circling either 1 or 2 on an answer sheet. The participants were told that many of the matches would be difficult but that they should always respond with their best guess. The experiment lasted about $1 \mathrm{~h}$ for each participant.

\section{Results and Discussion}

Means for the 10 speakers for both presentation conditions can be seen in Figure 1. Pooling over participants and speakers, the mean percent correct for the dynamic condition was $69.1 \%$ ( $S E=5.3 \%)$, whereas the mean percent correct for the static condition was $50.4 \%$ (3.3\%). The 10 speaker means for the dynamic condition ranged from $52.2 \%$ to $81.7 \%$, and the speaker means for the static condition ranged from $41.6 \%$ to $60.0 \%$. An analysis of variance (ANOVA) was conducted on the factors of presentation condition (static vs. dynamic) and speaker (1-10). This ANOVA revealed a significant effect of presentation con- 


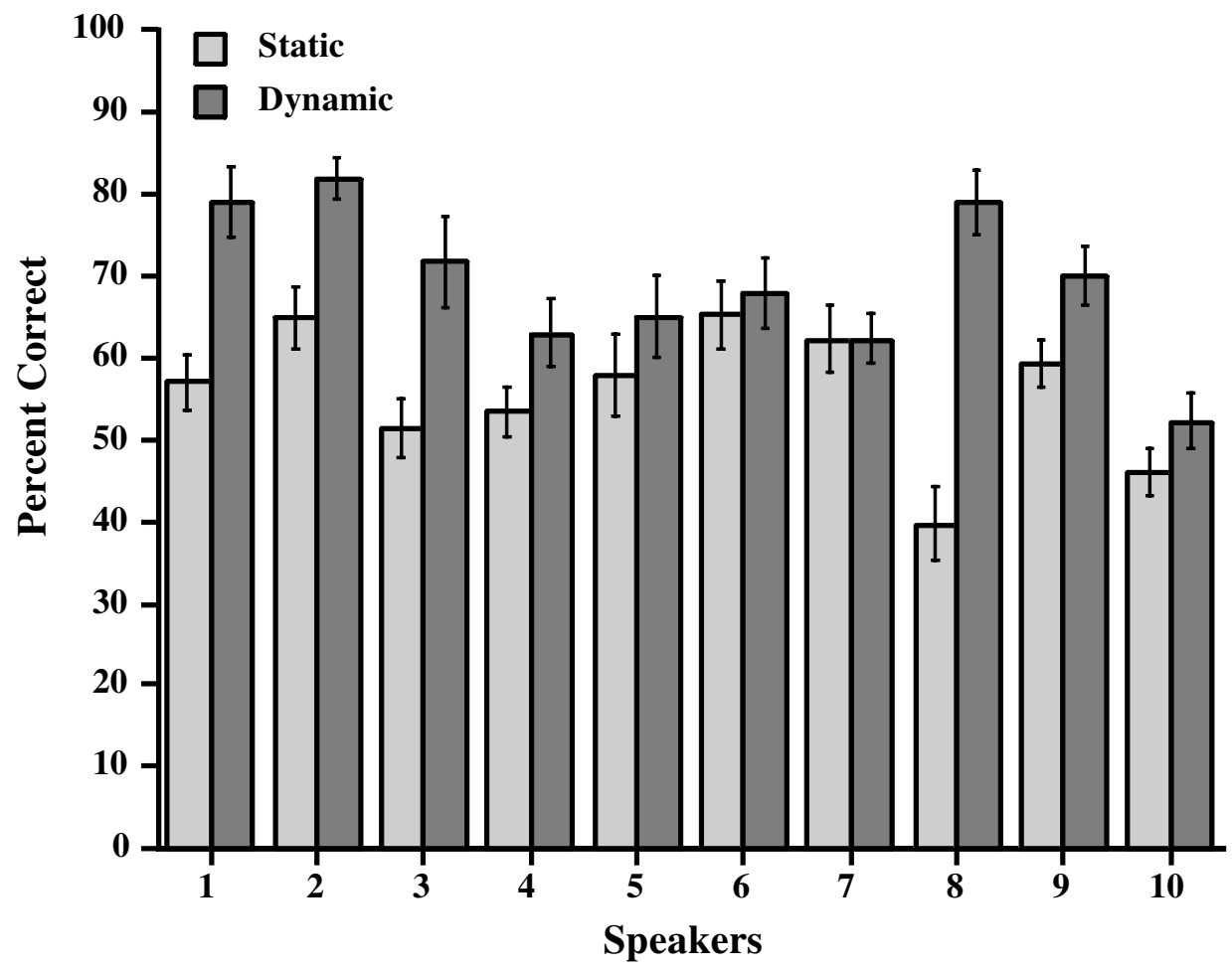

Figure 1. Mean speaker (and standard error) values for the static and dynamic conditions of Experiment 1.

dition $[F(1,180)=111.82, p<.0001]$ and of speaker $[F(9,180)=3.79, p=.0002]$, as well as an interaction of presentation conditionand speaker $[F(9,180)=4.06, p<.0001]$. Unpaired $t$ tests revealed that performance on the dynamic condition was significantly better than that on the static for 8 out of the 10 speakers $(p<.05)$.

Analyses (one-sample $t$ tests) were also conducted to determine under which conditions the participants performed at better than chance levels. Because judgments consisted of making matches between a pair of point-light stimuli, chance performance was set at $50 \%$ correct. For the dynamic condition, the participants correctly matched 9 of the 10 speakers at better than chance levels. For the static condition, the participants correctly matched 2 of the 10 speakers at better than chance levels $(p<.05)$.

Finally, a regression analysis was conducted on the speaker means for the dynamic condition with the speaker means of the static condition. No significant relationship was found between the patterning of these scores $[r=$ $.039 ; F(1,8)=0.12, p=.92$ ], indicating that the speakers most successfully matched in the dynamic condition were not the same as the speakers best matched in the static condition. This could suggest that the strategies and/or information used in the two conditions differed.

These results show that the participants were able to match an articulating point-light face to its articulating fully illuminated image at better than chance levels. Also, the fact that the participants were significantly better at making these matches in the dynamic condition than in the static condition could mean that this ability was not based on whatever static/structural information still remained in the point-light images. Potentially, these results could be interpreted as evidence that participants can match faces on the basis of isolated articulatory information.

However, before drawing this conclusion, another interpretation of our results must be examined. The dynamic and the static stimuli differed in another characteristic besides portraying dynamic articulation. Specifically, although both the dynamic and the static presentations each lasted $3 \mathrm{sec}$, the static stimuli were each composed of one image frame, whereas the dynamic stimuli were each composed of 90 image frames $(3 \mathrm{sec} \times 30$ frames per second [fps]). Thus, although the advantage of the dynamic stimuli might lie with the portrayal of dynamic articulatory information, it could simply be based on the dynamic presentations' containing more, and/or possibly more salient, static images. Although care was taken to select the same neutral mouth position across fully illuminated and pointlight images for the static stimuli, it could be that the other static images_or simply, more static images-making up the dynamic stimuli facilitated matching.

This issue was considered by Lander et al. (1999) in their dynamic face recognition study. To ensure that their observed dynamic stimulus advantage was not based on an unequal number of frames, they implemented methods to equalize the presented frames across dynamic and con- 
trol conditions. These methods involved reducing the frame rate in the dynamic condition from $25 \mathrm{fps}$ (European format) to $10 \mathrm{fps}$. Although this frame rate still portrayed movement, it made the task of equating the frames across dynamic and control conditions much easier.

In Experiment 2, we adopt a similar technique for equating the frames across dynamic and control conditions. In Experiment 2, each of the 100 dynamic stimuli (both fully illuminated and point-light) from Experiment 1 were reedited so that every 2 out of 3 frames were removed. For these reduced dynamic test stimuli, the remaining frames were displayed for an extra $2 \mathrm{fps}(100 \mathrm{msec}$ total $)$. This produced reduced dynamic stimuli composed of frames that changed at a rate of $10 \mathrm{fps}$, so that each sentence was made up of 30 total frames $(10 \mathrm{fps} \times 3 \mathrm{sec})$. This technique produced dynamic stimuli that looked somewhat less smooth but still conveyed articulatory information. (Informal pilot experiments showed that the sentence could be lip-read with comparable accuracy [see also Blokland \& Anderson, 1998; Vitkovitch \& Barber, 1994].) For Experiment 2, two control conditions were also tested. In the jumbled frame condition, the 30 frames of each pointlight and fully illuminated face sentence were presented in random order. In the staggered frame condition, the 30 frames of each sentence were played in the correct order, but the frames' presented durations varied randomly (e.g., Lander et al., 1999). Thus, the two control conditions of Experiment 2 were equated to the reduced dynamic condition in actual frames presented, and whereas the jumbled frame condition also equated frame duration $(100 \mathrm{msec})$, the staggered frame condition equated the ordering of the frames. These control conditions were designed to ensure that any difference in observed performance could not be attributed to static frame information, whether these static frames were equated in duration or ordering. ${ }^{1}$ If the dynamic movement information available in the reduced dynamic condition facilitates face matching, performance in that condition should be superior to performance in the jumbled frame or the staggered frame conditions.

\section{EXPERIMENT 2}

\section{Method}

Participants. Twenty-seven undergraduates were given class credit for their participation. Their ages ranged from 18 to 24 years. None of these participants had participated in Experiment 1.

Materials. The new reduced dynamic stimuli used in Experiment 2 were derived from the dynamic stimuli of Experiment 1 . The digitally captured sentences from Experiment 1 were manipulated, using the program Adobe Premiere. For each sentence, two out of every three frames were deleted (starting with Frames 2 and 3). In their place, the first of every three frames was repeated twice. This produced new dynamic sentence presentations composed of 10 sets of three repeated frames. Thus, the images changed at a rate of $10 \mathrm{fps}$, rather than the usual $30 \mathrm{fps}$. This frame deletion technique was applied to all of the fully illuminated and point-light stimuli to produce 100 ordered frame sentences. As in Experiment 1, the new dynamic sentences were arranged so that a fully illuminated utterance of a given speaker was presented first, followed by two point-light utter- ances. Again, all combinations were arranged in two orders, producing a total of 180 randomized triads (10 participants $\times 9$ matches $\times 2$ orderings). Again, the sentences were each $3 \mathrm{sec}$ long, with ISIs of $1 \mathrm{sec}$ between utterances in a triad and $3 \mathrm{sec}$ between triads.

The jumbled frame stimuli were derived from the reduced dynamic stimuli, using the Adobe Premiere program. For each sentence, the 10 sets of three repeated frames were placed in random order. This random order was derived with the constraint that no set appeared immediately after the set that preceded it in the ordered frame stimuli (no 2 sets of frames had correct adjacent order). The same random order of frame sets was applied to all the sentences (both the fully illuminated and the point-light) to produce 100 jumbled frame sentences. These sentences were arranged in 180 triads, using the same random ordering as that applied to the ordered frame stimuli.

The staggered frame stimuli were also derived from the reduced dynamic stimuli. For each sentence, the 10 different frames were presented once or were repeated in clusters of $3,5,7$, or 9 . These clusters were presented in the same (correct) order as those of the new dynamic stimuli. As in the other conditions, the total duration of each sentence was $3 \mathrm{sec}$, and the average frame rate was $10 \mathrm{fps}$. However, for the staggered frame condition, each frame was shown for $30,90,150,210$, or $360 \mathrm{msec}$. For each sentence, the duration of each consecutive frame varied randomly between these five values, with the constraint that the total duration should be equal to $3 \mathrm{sec}$. These random values were the same for all the sentences (both pointlight and fully illuminated). Thus, the resultant staggered stimuli maintained the same frames, presented in the same order, as the new dynamic stimuli but, because of the varied timing, did not retain the same dynamic information. The resultant images portrayed movement that looked somewhat jerky (Lander et al., 1999).

All other features of the stimuli were the same as those in Experiment 1 .

Procedure. Nine of the participants were randomly assigned to the reduced dynamic condition, 9 were assigned to the jumbled frame condition, and 9 were assigned to the staggered frame condition. All other aspects of the procedure were the same as those in Experiment 1 , including the stimulus presentation methodology, instructions, and response task. The experiment took about $1 \mathrm{~h}$ for each participant.

\section{Results and Discussion}

Means for the 10 speakers for the three presentation conditions can be seen in Figure 2. Pooling over participants and speakers, the mean percent correct for the reduced dynamic condition was $72.9 \%$ (3.3\%), whereas the mean percent correct for the jumbled frame condition was $55.4 \%$ (4.9\%), and the percent correct for the staggered frame condition was $60.0 \%(7.9 \%)$. The 10 speaker means for the reduced dynamic condition ranged from $67.3 \%$ to $78.4 \%$, the speaker means for the jumbled frame condition ranged from $48.8 \%$ to $61.7 \%$, and the speaker means for the staggered frame condition ranged from $48.7 \%$ to $74.6 \%$. An ANOVA was conducted on the factors of presentation condition (reduced dynamic, jumbled, staggered) and speaker (1-10). This ANOVA revealed a significant effect for presentation condition $[F(2,240)=33.62, p<.0001]$ and for speaker $[F(9,240)=2.23, p=.02]$, but not for the interaction of presentation condition and speaker $[F(18,240)=$ $0.887, p>$.05]. Post hoc Scheffé tests conducted on the levels of presentation condition revealed a significant difference between the reduced dynamic and the jumbled con- 


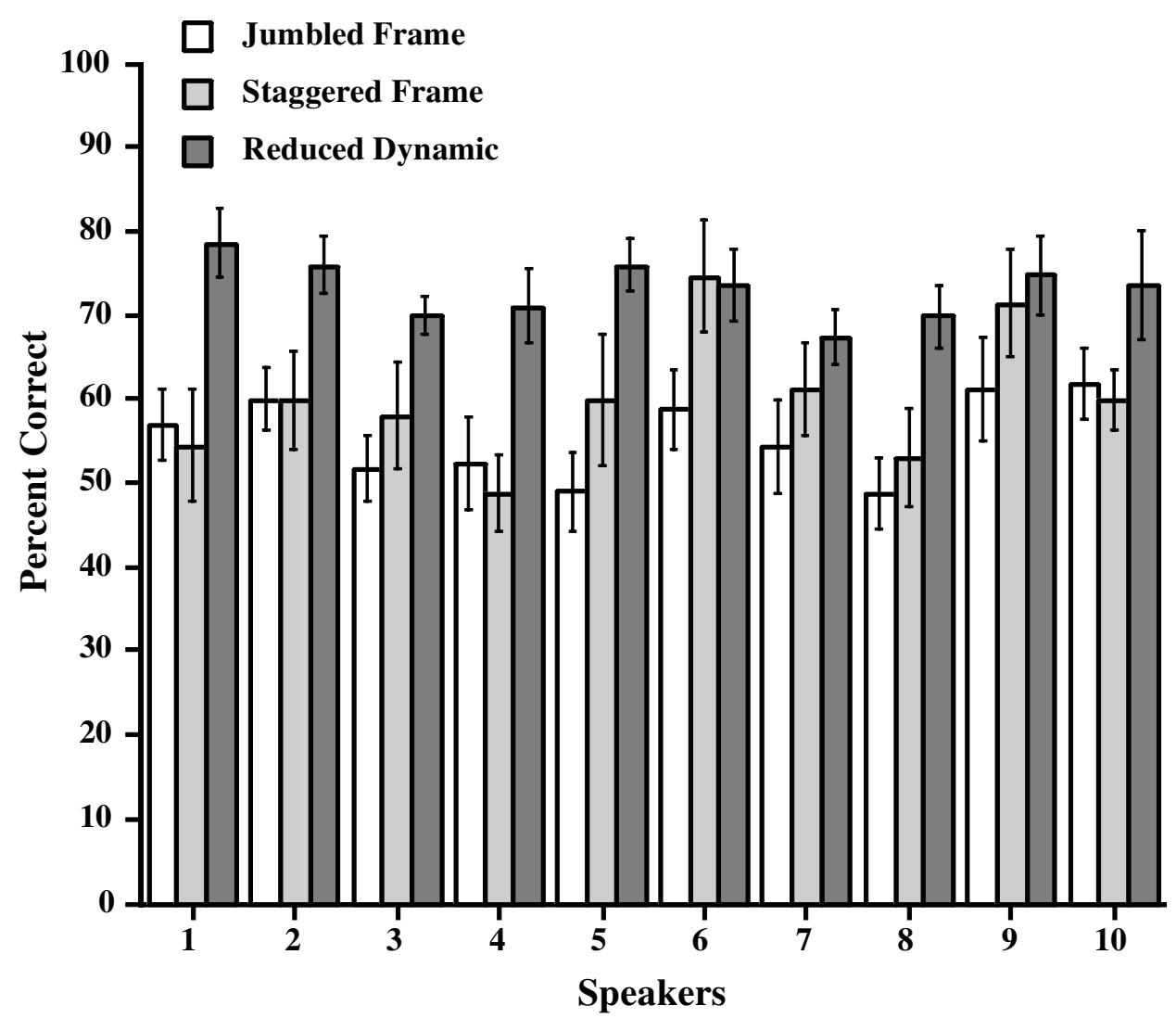

Figure 2. Mean speaker (and standard error) values for the jumbled, staggered, and reduced dynamic frame conditions of Experiment 2.

ditions $[F(2,240)=17.52, p<.0001]$, as well as between the reduced dynamic and the staggered conditions $[F(2,240)=$ $12.92, p<.0001]$, but not between the jumbled and the staggered conditions $[F(2,240)=-4.6, p=.13]$.

Analyses (one-sample $t$ tests) were also conducted to determine under which conditions the participants performed at better than chance levels. As before, chance performance was set at $50 \%$ correct. For the reduced dynamic condition, the participants correctly matched all 10 speakers at better than chance levels. For the jumbled frame condition, the participants correctly matched 2 of the 10 speakers at better than chance levels, and for the staggered frame condition, 3 of the 10 speakers were matched at better than chance levels $(p<.05)$.

As in Experiment 1, a regression analysis was conducted on the speaker means for the reduced dynamic condition with the speaker means of the control conditions. For the jumbled frame condition, no significant relationship was found between the patterning of these scores $[r=.405$; $F(1,8)=1.57, p=.245]$. There was also no correlation between the reduced dynamic condition and the staggered condition speaker scores $[r=.164 ; F(1,8)=0.221, p=$ .651]. A final correlation test was conducted between the speaker scores of the jumbled frame and the staggered frame conditions; although this test was also not significant at the $p<.05$ level, there was an observable trend toward a positiverelationship $[r=.552 ; F(1,8)=3.5, p=.098]$. These results could suggest that the strategies and/or the information used in the dynamic condition were different from those used in the jumbled and staggered control conditions. At the same time, the observed trend could suggest that similar strategies/information were used during the two control conditions. Future research can be conducted to examine this question.

The results of Experiment 2 suggest that the advantage of the dynamic condition observed in Experiment 1 was not a result of unequal frames between dynamic and static stimuli. In Experiment 2, the specific frames were the same in the reduced dynamic and the two control conditions. In the jumbled frame condition, the frames were each presented for the same duration as in the reduced dynamic condition $(100 \mathrm{msec})$. In the staggered frame condition, the order of the frames was the same as that in the reduced dynamic condition. Thus, it can be concluded that the difference in observed performance cannot be attributed simply to static frame information, whether these static frames are equated in duration, or ordering. In addition, the superior performance in the reduced dynamic condition ver- 
sus the staggered frame condition (despite the same frame order) makes it unlikely that the advantage of the dynamic conditions was due to a general Gestalt "filling-in" of points through interpolation between correctly ordered frames. Instead, it is likely that the reduced dynamic condition allowed for appropriate articulatory dynamic information to be conveyed, whereas the jumbled and the staggered frame conditions did not. These results provide stronger evidence that facial dynamics information can be used for speaker matching.

\section{GENERAL DISCUSSION}

These results add to the recent literature showing that movement can facilitate face recognition when images are degraded (Christie \& Bruce, 1998; Knight \& Johnston, 1997; Lander et al., 1999). The results augment the previous findings in three ways. First, for our results, the enhancing nature of facial movement was observed with a point-light image degradation technique. Although Valentine and Bruce (1985) have shown initial evidence for this fact, their comparison stationary stimuli were composed only of individual static images. Our Experiment 2 confirmed that the advantage of the moving point-light stimuli was based on its ordered dynamic information, and not simply on a greater number of static images. In confirming the informativeness of point-light stimuli for face matching, our findings add to research that has used other stimulus degradation techniques. It is likely that the point-light methodology produces images that maintain even less featural/structural information than do the previously used techniques of image negation, thresholding, and inversion (Knight \& Johnston, 1997; Lander et al., 1999; Lander et al., 2001), a point made by Knight and Johnston. At the very least, our data support the claim of Lander et al. (1999) that movement provides enhancing information for any "nonoptimal" viewing condition and is not limited to specific image defects.

Second, our experiments show that the facilitory nature of movement is maintained in a matching task. Prior research has used tasks involving recognition of famous faces (Lander et al., 1999) or personally familiar faces (Bruce \& Valentine, 1988), as well as recognition of unfamiliar faces made familiar through pretest learning (Christie \& Bruce, 1998; Pike, Kemp, Towell, \& Phillips, 1997; Schiff, Banka, \& De Bordes Galdi, 1986). The task demands of matching might be fewer than the demands for longer term memory. Reduced task demands might allow observers to more easily detect subtle informational differences between stimuli. The fact that a dynamic stimulus advantage still exists with the matching task supports the generality of the moving advantage.

Finally, our findings add to the recent literature in showing that the visual information provided primarily by speech articulation can facilitate face recognition. The theoretical implications of this finding will be discussed below. However, it should be noted that the previous research showing a moving-stimulus facilitation incorporated more extreme face and head movements (Christie \& Bruce, 1998; Pike et al., 1997; Schiff et al., 1986; Valentine \& Bruce, 1985). Our findings show that the relatively subtle movements of speech articulation also provide information that enhances face perception.

\section{Information in a Moving Face}

Following the discussion of Christie and Bruce (1998), there are three general ways in which dynamic face displays might be informative. First, seeing a moving face might provide more static face information than is available in a single static image. Although this could be a reason other moving face displays are enhancing, it is unlikely to be the primary reason for the enhancementobserved in the present study. In equating the static information across displays, Experiment 2 shows that there is information contained in a moving display that cannot be captured by simply displaying its static frames (see also Pike et al., 1997).

Second, Christie and Bruce (1998) propose that movement might provide greater information about the three dimensional(3-D) structure of a face. This structure-frommotion information has been used to explain nonface point-light display recognition (e.g., Braunstein, Hoffman, \& Pollick, 1990; Hildreth, Grzywacz, Adelson, \& Inada, 1990; Johansson, 1973, Pollick, 1997; Ullman, 1979) for both rigid and nonrigid motions, the latter of which is likely more relevant for facial movements. Third, Christie and Bruce propose that a characteristic style of facial movement might be informative for face recognition. These transformational invariants (Gibson, 1979) could be conveyed in the point-light images. It is this last category of information that likely encompasses articulatory style/idiolectic information.

The present experiments cannot resolve whether motion acts more to add 3-D structural face information or to convey face-specific style of motion. However, this issue has been addressed elsewhere in the literature. For example, Knight and Johnston (1997) interpret their results as supportive that movement provides additional 3-D information about a face. They used negative image degradation, which has been considered to maintain 2-D image configural and featural information while inhibiting recognition. Negating an image reduces much of the shading and brightness contrast information useful for extracting 3-D structure (e.g., Johnston, Hill, \& Carman, 1992). Knight and Johnston argue that the 3-D structure most distorted in negating face images is that associated with configural face information - that is, the information contained in facial shape and the spatial relation between facial features. In fact, it is the configural information contained in a face that is thought to be made unavailable when a face is inverted, thereby making inverted face recognition disproportionately difficult (e.g., Carey \& Diamond, 1977; Scapinello \& Yarmey, 1970; Yin, 1969; and see Valentine, 1988 , for a review). Knight and Johnston found that facial 
motion enhanced recognition of negative image faces that were upright, but not those that were inverted. One interpretation of this finding is that motion primarily provides additional 3-D information that embellishes configural dimensions-dimensions that are most available in upright faces.

However, the recent findings of Lander et al. (1999), as well as those of Lander et al. (2001), show that movement can enhance recognition of inverted faces as well. Lander and her colleagues interpret these findings as support that the enhancing effect of motion must lie in more than simply reinstating 3-D information. Potentially, motion enhances face recognition under all of these conditions because it also conveys facial movements that are characteristic of an individual. Lander et al. (1999) further suggest that the use of characteristic motion could explain their result that recognition was hindered by distortions of the movement dynamics (i.e., slowing or changing the rhythm of the video presentations).

If, in fact, facial movements specific to an individualcan be informative for recognition, it is likely that the characteristic movements in our displays took the form of speech articulation. The actors of our stimuli were constrained to keep their heads still and only to move their face in such a way as to articulate the sentence (with no expression). Although points were placed on facial surfaces outside the mouth area, most of the movement observable in our displays occurred in the mouth region. This is not to suggest that other types of nonspeech face movements cannot be informative about identity. Rather, it is simply argued that the information contained in our stimuli likely took the form of speech articulation.

What aspects of articulatory style might have been informative for speaker matching in our experiments? At this time, we can only offer speculation. However, it should be noted that whatever dimensions provided the basis for matching each speaker, these dimensions were maintained over multiple utterances of the sentence. For every speaker, the nine different point-light utterances and the one fully illuminated utterance were derived from separate video recordings. These utterances were typically recorded over a 3-h period (allowing time to apply the point-light preparation). Thus, this idiosyncratic articulatory information was specific neither to a single utterance nor to a series of adjacent utterances for a speaker. These facts suggest that whatever dimensions were informative, they were likely indicative of a speaker's general style of talking. If speakers' general idiolectic style information is salient, face matching should be possible across different sentential material. It also follows that observers who have extensive personal familiarity with the speakers should be able to perform identification (vs. matching) judgments, using point-light speech information. Research is currently underway in our laboratory to test these predictions.

\section{Common Visual Information for Speech and Speaker Recognition}

Our results provide an initial demonstration that isolated, dynamic visible speech information can be informative for face recognition. However, our results do not suggest that only the facial movements related to speech articulation are informative for identification. It could very well be that nonspeech face movements, including those associated with emotional expression or even chewing, might also inform about identity. Still, the finding that visible speech movements can provide information for face recognition has interesting theoretical implications. As has been mentioned, the functions of visual speech and face perception are usually considered to be very separate. This dissociation has also been considered to hold for the informational basis of the functions. Quite recently, however, evidence has appeared that common facial image information can influence both visual speech and face perception functions. As was stated above, research has shown that upright facial context information can influence both functions. Perceptual phenomena, such as facial inversion and Margaret Thatcher effects (e.g., Thompson, 1980; Valentine, 1988), considered to be specific to face perception have been shown to analogously influence visual speech perception (Green, 1994; Jordan \& Bevan, 1997; Massaro \& Cohen, 1996; Rosenblum et al., 2000). Although dynamic information was used, the present findings add to the research showing that common information can bear on both functions.

The accumulating findings that common information can be used for both functions could have more far-reaching implications for theories of face and visual speech perception. It could be that the contingencies observed of visual speech on face familiarity (e.g., Walker et al., 1995; Yakel et al., 2000) might be based on this use of common information. A similar suggestion has been offered by Remez and his colleagues with regard to auditory speech. Recall that Remez et al. (1997) found that the isolated phonetic speech information contained in sinewave sentences can inform about talker identity. In discussing their results, these authors suggest that phonetic attributes of the signal might be used for both linguistic and talker recognition processes. They further suggest that the observed contingencies of lexical recovery on speaker-specific attributes might be accomplished, in part, by a common phonetic analysis. Thus, a phonetic account could rationalize many of the auditory speech contingency effects discussed above.

Analogously, findings that common visual speech information can be used for both visual speech perception and face matching might help to explain the observed contingencies between face and visual speech functions. The contingencies might be a consequence of extracting visible articulatory dimensions that incidentally provides speaker-specific information. The present findings motivate further research into this possibility.

\section{REFERENCES}

Assal, G., Aubert, C., \& Buttet, J. (1981). Asymétrie cérébrale et reconnaissance de la voix. Revue Neurologique, 137, 255-268.

Assal, G., Zander, E., Kremin, H., \& Buttet, J. (1976). Discrimination des voix lors de lésions du cortex cérébral. Archives Suisses de Neurologie, 119, 307-315.

Barber, P. J., Vitkovitch, M., Wallbott, H., Ellgring, H. E., \& Kotthaus, B. (1990). Recommendations for screen display parame- 
ter settings for a videolink from a human performance perspective. European Commission, RACE-Project, R1086 TELEMED.

BAssili, J. N. (1978). Facial motion in the perception of faces and of emotional expression. Journal of Experimental Psychology: Human Perception \& Performance, 4, 373-379.

Berry, D. S. (1990). What can a moving face tell us? Journal of Personality \& Social Psychology, 58, 1004-1014.

BERRY, D. S. (1991). Child and adult sensitivity to gender information in patterns of facial motion. Ecological Psychology, 3, 349-366.

Blokland, A., \& Anderson, A. H. (1998). Effect of low frame-rate video on intelligibility of speech. Speech Communication, 26, 97-103.

Braunstein, M. L., Hoffman, D. D., \& Pollick, F. E. (1990). Discriminating rigid from nonrigid motion: Minimum points and views. Perception \& Psychophysics, 47, 205-214.

Bricker, P. D., \& PruZansky, S. (1966). Effects of stimulus content and duration on talker identification. Journal of the Acoustical Society of America, 40, 1441-1449.

BRUCE, V. (1988). Recognising faces. Hove, U.K.: Erlbaum.

Bruce, V., \& VAlentine, T. (1988). When a nod's as good as a wink: The role of dynamic information in facial recognition. In M. M. Gruneberg, P. E. Morris, \& R. N. Sykes (Eds.), Practical aspects of memory: Current research and issues: Vol. 1. Memory in everyday life (pp. 169-174). New York: Wiley.

Bruce, V., \& Young, A. (1986). Understanding face recognition. British Journal of Psychology, 77, 305-327.

CAmpbell, R. T., Landis, T., \& Regard, M. (1986). Face recognition and lip-reading: A neurological dissociation. Brain, 109, 509-521.

Carey, S., \& Diamond, R. (1977). From piecemeal to configurational representation of faces. Science, 195, 312-314.

Christie, F., \& Bruce, V. (1998). The role of dynamic information in the recognition of unfamiliar faces. Memory \& Cognition, 26, 780-790.

Church, B. A., \& Schacter, D. L. (1994). Perceptual specificity of auditory priming: Implicit memory for voice intonation and fundamental frequency. Journal of Experimental Psychology: Learning, Memory, \& Cognition, 20, 521-533.

Craik, F. I., \& KIRSNER, K. (1974). The effect of speaker's voice on word recognition. Quarterly Journal of Experimental Psychology, 26, 274-284.

ELLIS, H. D. (1989). Processes underlying face recognition. In R. Bruyer (Ed.), Neuropsychology of face perception and facial expression (pp. 471-478). Hillsdale, NJ: Erlbaum.

FoDor, J. A. (1983). Modularity of mind. Cambridge, MA: MIT Press, Bradford Books.

GiBSON, J. J. (1979). The ecological approach to visual perception. Boston: Houghton-Mifflin.

Goldschen, T. M. (1993). Continuous automatic speech recognition by lipreading. Unpublished doctoral dissertation, George Washington University.

Green, K. P. (1994, June). The influence of an inverted face on the McGurk effect. Poster presented at the annual meeting of the Acoustical Society of America, Cambridge, MA. Journal of the Acoustical Society of America, 95, 3014 [Abstract].

Green, K. P., \& Gerdman, A. (1995). Cross-modal discrepancies in coarticulation and the integration of speech information: The McGurk effect with mismatched vowels. Journal of Experimental Psychology: Human Perception \& Performance, 21, 1409-1426.

Hildreth, E. C., Grzywacz, N. M., Adelson, E. H., \& Inada, V. K. (1990). The perceptual buildup of three-dimensional structure from motion. Perception \& Psychophysics, 48, 19-36.

Johansson, G. (1973). Visual perception of biological motion and a model for its analysis. Perception \& Psychophysics, 14, 201-211.

Johnston, A., Hill, H., \& Carman, N. (1992). Recognising faces: Effects of lighting direction, inversion, and brightness reversal. Perception, 21, 365-375.

JoRDAN, T. R. \& BEvAN, K. (1997). Seeing and hearing rotated faces: Influences of facial orientation on visual and audio-visual speech recognition. Journal of Experimental Psychology: Human Perception \& Performance, 23, 388-403.

Knight, B., \& Johnston, A. (1997). The role of movement in face recognition. Visual Cognition, 4, 265-273.

Lander, K., Christie, F., \& Bruce, V. (1999). The role of movement in the recognition of famous faces. Memory \& Cognition, 27, 974-985.
Lander, K., Rosenblum, L. D., \& Bruce, V. (2001). Recognizing "Thatcherized" faces: Influences of inverted and dynamic presentations. Manuscript in preparation.

Liberman, A. M., \& Mattingly, I. G. (1985). The motor theory of speech perception revised. Cognition, 21, 1-36.

Massaro, D. W., \& Cohen, M. M. (1996). Perceiving speech from inverted faces. Perception \& Psychophysics, 58, 1047-1065.

McGRATH, M. (1985). An examination of cues for visual and audio-visual speech perception using natural and computer-generated faces. Unpublished doctoral thesis, University of Nottingham.

McGurK, H., \& MacDonald, J. W. (1976). Hearing lips and seeing voices. Nature, 264, 746-748.

Montgomery, A. A., \& JACKson, P. L. (1983). Physical characteristics of the lips underlying vowel lipreading performance. Journal of the Acoustical Society of America, 73, 2134-2144.

Mullennix, J. W., Pisoni, D. B., \& Martin, C. S. (1989). Some effects of talker variability on spoken word recognition. Journal of the Acoustical Society of America, 92, 1085-1099.

Ny gaArd, L. C., Sommers, M. S., \& Pisoni, D. B. (1994). Speech perception as a talker-contingent process. Psychological Science, 5, 42-46.

Palmeri, T. J., Goldinger, S. D., \& Pisoni, D. B. (1993). Episodic encoding of voice attributes and recognition memory for spoken words. Journal of Experimental Psychology: Learning, Memory, \& Cognition, 19, 309-328.

Petajan, E. D. (1984). Automatic lipreading to enhance speech recognition. In Proceedings of the IEEE Communications Society, Piscataway, NJ: IEEE Press.

Pike, G. E., Kemp, R. I., Towell, N. A., \& Phillips, K. C. (1997). Recognizing moving faces: The relative contribution of motion and perspective view information. Visual Cognition, 4, 409-437.

PoLLICK,F. E. (1997). The perception of motion and structure in structurefrom-motion: Comparisons of affine and Euclidean formulations. $\mathrm{Vi}$ sion Research, 37, 447-466.

Remez, R. E., Fellowes, J. M., \& Rubin, P. E. (1997). Talker identification based on phonetic information. Journal of Experimental Psychology: Human Perception \& Performance, 23, 651-666.

Rosenblum, L. D., Johnson, J. A., \& Mahmood, C. (2001). Kinematic features for visual speech perception. Manuscript in preparation.

Rosenblum, L. D., Johnson, J. A., \& Saldaña, H. M. (1996). Visual kinematic information for embellishing speech in noise. Journal of Speech \& Hearing Research, 39, 1159-1170.

Rosenblum, L. D., \& Saldaña, H. M. (1996). An audiovisual test of kinematic primitives for visual speech perception. Journal of Experimental Psychology: Human Perception \& Performance, 22, 318-331.

Rosenblum,L. D., \& SAldaÑa, H. M. (1998). Time-varying information for visual speech perception. In R. Campbell, B. Dodd, \& D. Burnham (Eds.), Hearing by eye: Pt. 2. The psychology of speechreading and audiovisual speech (pp. 61-81). Hillsdale: NJ: Erlbaum.

Rosenblum, L. D., Yakel, D. A., \& Green, K. G. (2000). Face and mouth inversion affects on visual and audiovisual speech perception. Journal of Experimental Psychology: Human Perception \& Performance, 26, 806-819.

Saldaña, H. M., \& Rosenblum, L. D. (1994). Voice information in auditory form-based priming [Abstract]. Journal of the Acoustical Society of America, 95, 2870.

SCAPINELlo, K. F., \& YARMEY, A. D. (1970). The role of familiarity and orientation in immediate and delayed recognition of pictorial stimuli. Psychonomic Science, 21, 329-331.

Schiff, W., Banka, L., \& De Bordes Galdi, G. (1986). Recognizing people seen in events via dynamic "mugshots." American Journal of Psychology, 99, 219-231.

Schweinberger, S. R., \& Soukup, G. R. (1998). Asymmetric relationships among perceptions of facial identity, emotion, and facial speech. Journal of Experimental Psychology: Human Perception \& Performance, 24, 1748-1765.

Sheffert, S. M., \& Fowler, C. A. (1995). The effects of voice and visible speaker change on memory for spoken words. Journal of Memory \& Language, 34, 665-685.

Summerfield, Q., \& MCGRATh, M. (1984). Detection and resolution of audio-visual incompatibility in the perception of vowels. Quarterly Journal of Experimental Psychology, 36A, 51-74. 
Thompson, P. (1980). Margaret Thatcher: A new illusion. Perception, 9 , 483-484.

Ullman, S. (1979). The interpretation of visual motion. Cambridge, MA: MIT Press.

VALENTINE, T. (1988). Upside-down faces: A review of the effect of inversion upon face recognition. British Journal of Psychology, 79, 471-491.

Valentine, T., \& Bruce, V. (1985). What's up? The Margaret Thatcher illusion revisited. Perception, 14, 515-516.

Van Lancker, D., Cummings, J. L., Kreiman, J., \& Dobkin, B. H. (1988). Phonagnosia: A dissociation between familiar and unfamiliar voices. Cortex, 24, 195-209.

VITKOVITCH, M., \& BARBER, P. (1994). Effect of video frame rate on subjects' ability to shadow one of two competing verbal passages. Journal of Speech \& Hearing Research, 37, 1204-1210.

Walker, S., Bruce, V., \& O'Malley, C. (1995). Facial identity and fa- cial speech processing: Familiar faces and voices in the McGurk effect. Perception \& Psychophysics, 57, 1124-1133.

Yakel, D. A., Rosenblum, L. D., \& Fortier, M. A. (2000). Effects of talker variability on speechreading. Perception \& Psychophysics, 62, 1405-1412.

YIN, R. K. (1969). Looking at upside-down faces. Journal of Experimental Psychology, 81, 141-145.

\section{NOTE}

1. We thank two anonymous reviewers for suggesting this alternative interpretation.

(Manuscript received November 23, 1999; revision accepted for publication June 12, 2001.) 